\title{
Composição de macroinvertebrados associados a macrófitas aquáticas como parâmetro para avaliação da qualidade da água de um reservatório no semiárido baiano
}

\author{
Mailson Silva Santana ${ }^{*}$ \\ Celimarcos Bezerra dos Santos ${ }^{2}$ \\ Patrícia Maria Mitsuka ${ }^{1}$ \\ Universidade do Estado da Bahia, Departamento de Ciências Humanas, Campus VI \\ CEP 46.400-000, Caetité - BA, Brasil \\ ${ }^{1}$ Laboratório de Ecologia do Semiárido \\ ${ }^{2}$ Grupo de Pesquisa em Ecologia do Semiárido, Linha de Pesquisa em Ecologia Aquática \\ * Autor para correspondência \\ santanamailson4@gmail.com
}

Submetido em 30/11/2020

Aceito para publicação em 03/07/2021

\section{Resumo}

Macroinvertebrados associados a macrófitas aquáticas tornam-se mais abundantes, pois as características morfológicas dessas plantas contribuem para o aumento da sua colonização, diversidade e avaliação da qualidade ambiental. Esse estudo apresenta o levantamento da composição e riqueza de macroinvertebrados (Classe Insecta) associados a diferentes espécies de macrófitas aquáticas e avalia a qualidade da água do reservatório Tanque de Aroeiras, Caetité, Bahia. Macroinvertebrados foram coletados em bancos de macrófitas de diferentes biótipos (Nymphaea L.; Ludwigia L.; Polygonum ferrugineum Wedd.) e identificados no nível taxonômico de família. Foram determinados os índices Biological Monitoring Working Party System (BMWP') e Average Score per Taxa (BMWP-ASPT). O total de 7.120 espécimes foi registrado, com riqueza de 27 famílias, distribuídas nas ordens: Coleoptera, Diptera, Ephemeroptera, Hemiptera, Odonata e Trichoptera. Os coleópteros apresentaram maior riqueza; enquanto dípteros, maior dominância, sendo Chironomidae mais abundante. Tal fato pode estar associado à variedade de hábitos de vida aquático, o que contribui para uma maior disponibilidade de recursos alimentares. Os resultados do BMWP-ASPT classificaram a água desse reservatório como sendo de provável poluição moderada. Enfim, este estudo ressalta a importância de novas pesquisas para um maior conhecimento da comunidade de macroinvertebrados e sua aplicação na avaliação da qualidade da água desse reservatório.

Palavras-chave: Biomonitoramento; Insetos aquáticos; Reservatórios temporários

\section{Abstract}

Composition of macroinvertebrates associated with aquatic macrophytes as a parameter for assessing water quality in a reservoir in the semiarid region of Bahia. Macroinvertebrates associated with aquatic macrophytes have become more abundant since the plant's morphological characteristics contribute to an increase in colonization, diversity and environmental quality assessment. This study presents a survey of the composition and richness of macroinvertebrates (Insecta Class) associated with different species of aquatic macrophytes and 
evaluates the water quality of the Tanque de Aroeiras reservoir, Caetité, Bahia. Macroinvertebrates were collected from macrophyte banks of different biotypes (Nymphaea L.; Ludwigia L.; Polygonum ferrugineum Wedd.) and identified at the family taxonomic level. The Biological Monitoring Working Party System (BMWP) and Average Score per Rate (BMWP-ASPT) indices were determined. A total of 7,120 specimens were recorded, with a richness of 27 families, distributed in the following orders: Coleoptera, Diptera, Ephemeroptera, Hemiptera, Odonata and Trichoptera. Coleoptera showed greater richness, while dipterans showed greater dominance, with Chironomidae being more abundant. This fact may be associated with a variety of aquatic life habits that contribute to a greater availability of food resources. The results of the BMWP-ASPT classified the water in this reservoir as likely to be moderately polluted. All things considered, this study highlights the importance of new research for added knowledge regarding the macroinvertebrate community and its application in evaluating the water quality of this reservoir.

Key words: Aquatic Insects; Biomonitoring; Temporary reservoirs

\section{Introdução}

O reservatório Tanque de Aroeiras é um pequeno biossistema que abriga em seu limite e entorno uma grande diversidade de organismos que dependem diretamente de seus recursos. Esse tipo de reservatório é frequente na paisagem do semiárido brasileiro, sendo uma alternativa para combater e mitigar o efeito da seca, refletindo sua importância para o desenvolvimento da vida na região (PEREIRA NETO, 2017).

Classificados pelo seu tamanho, raramente inferior a $1 \mathrm{~mm}$ (MUGNAI et al., 2010; SILVEIRAMANZOTTI et al., 2016), os macroinvertebrados são um dos grupos mais representativos na fauna aquática por apresentar alta abundância, biodiversidade e ampla distribuição. Diversos grupos compõem a comunidade de macroinvertebrados aquáticos, como o subfilo Hexapoda, que representa o grupo mais diverso de animais dulciaquícolas, aquáticos e semiaquáticos, com mais de sessenta mil espécies. Dentro desse subfilo, a classe Insecta é dominante nos sistemas de água doce em termos de número de espécies, biomassa e produtividade (GULLAN; CRANSTON, 2012; FERREIRA-JÚNIOR et al., 2014).

Conforme o habitat, os macroinvertebrados apresentam ampla distribuição no ecossistema lêntico, mas, preferencialmente, encontram na zona litorânea características exclusivas que favorecem o desenvolvimento de sua comunidade (BUTAKKA et al., 2014). Presentes nessa região estão as macrófitas aquáticas, que, com diferenças morfológicas marcantes entre seus biótipos, contribuem para o aumento da complexidade e heterogeneidade de habitat e, respectivamente, para a abundância e a biodiversidade (TANIGUCHI; TOKESHI, 2004; THOMAZ; CUNHA, 2010). De forma geral, as macrófitas proporcionam maior oferta de abrigo contra predadores e alimento para a fauna local, elevando a biodiversidade e as relações interespecíficas, como entre macroinvertebrados e macrófitas aquáticas (ALBERTONI et al., 2007; THOMAZ; CUNHA, 2010). Essa interação biológica está condicionada à textura do substrato, à capacidade de retenção de matéria orgânica particulada e à presença de perifíton (POI DE NEIFF; NEIFF, 2006; SILVA; HENRY, 2013).

No entanto, nas últimas décadas, essa interação tem sido afetada em decorrência de ações antrópicas como: atividades de mineração, cultivo de terras agrícolas, utilização de agrotóxicos e despejo de esgoto doméstico e/ou industrial não tratado (CALLISTO et al., 2001b; FULAN et al., 2012). Poluentes oriundos dessas atividades são lançados diretamente ou lixiviados para os ambientes, afetando a fisiologia natural, a estrutura e a dinâmica dos ecossistemas (TUNDISI; TUNDISI, 2008). $\mathrm{O}$ aumento nas concentrações de fosfato e compostos nitrogenados favorece a superabundância de macrófitas aquáticas flutuantes, limitando o desenvolvimento da comunidade fitoplanctônica, bem como toda a estrutura trófica. Esse processo poderá conduzir a um estado de anoxia ambiental, afetando a saúde do ecossistema, ou seja, a qualidade da água (KLUMPP et al., 2002; BIUDES; CAMARGO, 2006; XAVIER et al., 2016; COLADELLO et al., 2020). 
Atualmente, existem diferentes metodologias para avaliação da qualidade da água, desenvolvidas como variações do sistema saprobiótico, em que índices são calculados sobre a presença de organismos indicadores que recebem valores correspondentes a sua tolerância à poluição (JUNQUEIRA et al., 2000; MONTEIRO et al., 2008). Por isso, a qualidade da água pode ser medida não somente pelos parâmetros físico-químicos limnológicos, mas também por parâmetros biológicos, através do levantamento de comunidades de fitoplâncton, macrófitas aquáticas, zooplâncton, microrganismos e bentos (CALLISTO; GONÇALVES, 2002; KLUMPP et al., 2002; BUSS et al., 2003; MONTEIRO et al., 2008; MUGNAI et al., 2010).

Dessa forma, entre os grupos que compõem a biodiversidade aquática, os macroinvertebrados são mais usados como bioindicadores por terem ampla distribuição, riqueza, abundância funcional e ciclo de vida relativamente longo. Além disso, possuem em sua maioria características ecológicas conhecidas, sendo relativamente sedentários, pois possuem mobilidade limitada e apresentam diferentes graus de sensibilidade a estresses ambientais (ROSENBERG; RESH, 1993; MORENO; CALLISTO, 2004; MONTEIRO et al., 2008; GULLAN; CRANSTON, 2012). Acrescentase, ainda, a ampla plasticidade que esses organismos apresentam, diferindo entre si na relação com os diferentes tipos de impactos ambientais: há desde organismos típicos de ambientes com pouca ou nenhuma poluição (por exemplo, ninfas de Plecoptera e larvas de Trichoptera), passando por organismos tolerantes (alguns Heteroptera e Odonata) até organismos resistentes (alguns Chironomidae da ordem Diptera) (ROSENBERG; RESH, 1993; CALLISTO et al., 2001b). Por fim, trata-se de organismos que respondem rapidamente às alterações nas condições locais do ambiente, além de permitir a detecção de estresses pontuais no tempo (ROSENBERG; RESH, 1993).

Com base nessas considerações, nosso estudo objetivou realizar o levantamento da composição e riqueza de macroinvertebrados da classe Insecta associados a diferentes espécies de macrófitas aquáticas, em Tanque de Aroeiras, Caetité - BA. Além disso, buscou avaliar a qualidade da água desse reservatório por meio de índices biológicos, utilizando como ferramenta as famílias de macroinvertebrados. Essa abordagem faz-se necessária, pois espera-se que, em condições ambientais específicas, como níveis diferenciados de poluição, os grupos mais resistentes desses animais possam dominar o ambiente aquático e, por outro lado, que os organismos mais sensíveis possam tornar-se raros ou extintos localmente (ABÍLIO et al., 2007).

\section{Material e Métodos}

\section{Área de estudo}

O trabalho foi realizado no Tanque de Aroeiras (1403'51'S e 42 $39^{\prime} 00^{\prime}$ 'O), zona rural que dista aproximadamente $21 \mathrm{~km}$ do município sede Caetité, sudoeste da Bahia (Figura 1).

Esse tanque apresenta uma extensão perimetral de 609,97 m e área de $23.452,29 \mathrm{~m}^{2}$, porém a porção que envolve o espelho de água é menor (Figura 1). Ressaltase que esses dados são compostos apenas pelas águas do período chuvoso, não havendo nenhuma outra fonte de água que abasteça o reservatório ao longo do ano. Além disso, tornou-se muito importante na dinâmica da comunidade rural, pois é utilizado para diversos fins, como irrigação de hortaliças, criação de animais, pesca e até mesmo para o consumo humano em períodos prolongados de estiagem.

O reservatório apresenta, em seu entorno, uma quantidade substancial de área desflorestada, sendo essa região ocupada e utilizada para agricultura familiar e pecuária. Além disso, quase toda a extensão da margem litorânea do Tanque de Aroeiras é provida da presença de macrófitas aquáticas (Figura 1).

O bioma na região é a Caatinga, apresentando áreas de transição com o Cerrado. O predomínio da vegetação xerófita compreende árvores e arbustos baixos, sendo a maioria dotada de espinhos e microfilia (PRADO, 2003). O clima é caracterizado como ameno, com média mensal de temperatura variando entre $19,3^{\circ} \mathrm{Ce} 22,2^{\circ} \mathrm{C}$ nos meses de julho e dezembro, respectivamente. Além desses dados climáticos, os valores registrados de precipitação podem chegar a $890 \mathrm{~mm}$ ao longo de um ano, sendo em 
FIGURA 1: Mapa de uso e ocupação do solo no entorno do reservatório Tanque de Aroeiras, Caetité, Bahia.

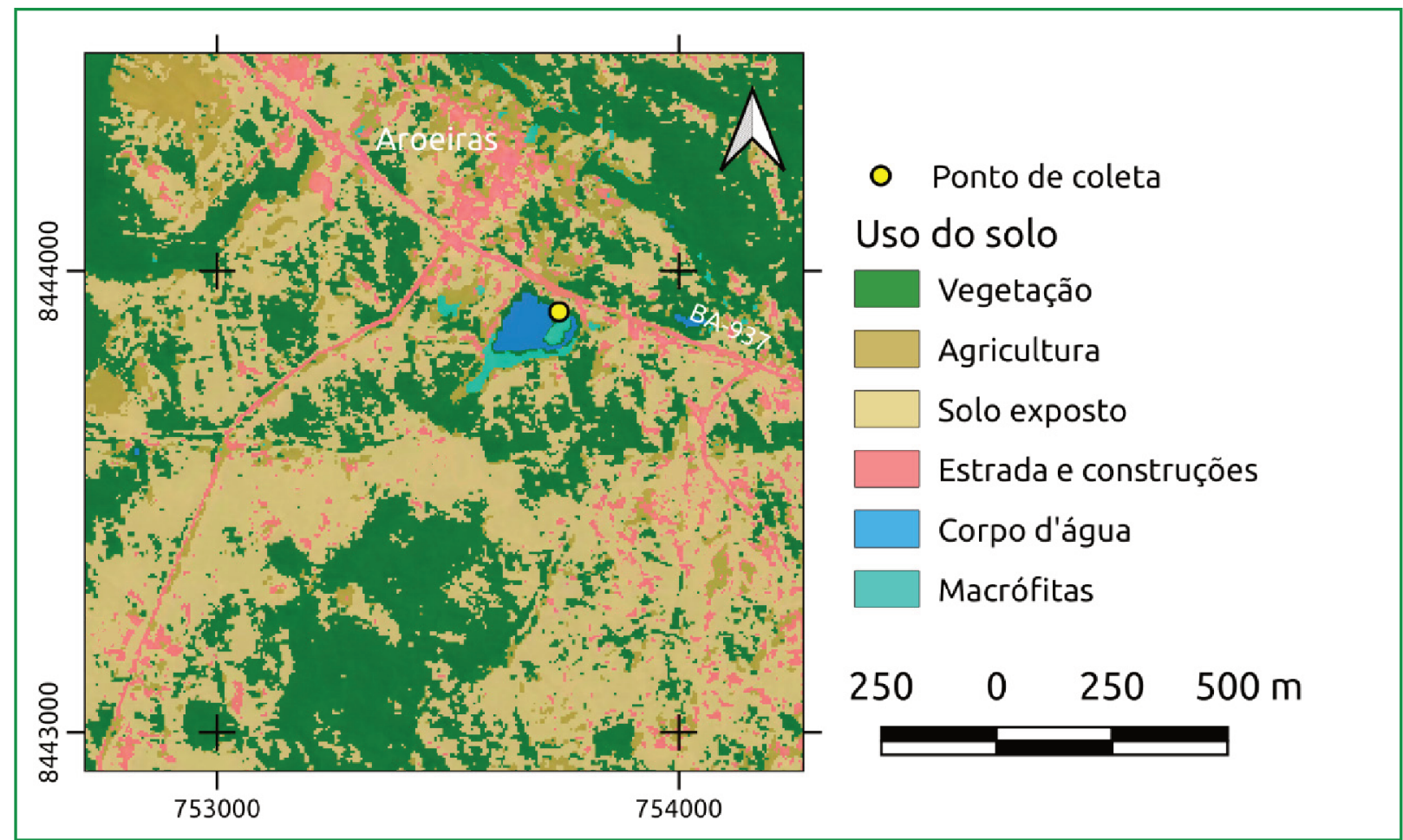

média o mês de agosto mais seco $(6 \mathrm{~mm})$ e o de dezembro o mais chuvoso (180 mm) (INMET, 2020).

\section{Procedimento metodológico}

Em agosto de 2018, período de estiagem, foi realizada a coleta em três (3) diferentes bancos de macrófitas, distando aproximadamente $3 \mathrm{~m}$ entre um banco e outro e até $1,5 \mathrm{~m}$ do banco para a margem. Conforme o maior predomínio no Tanque, e a facilidade de acesso, foram utilizados os seguintes bancos de macrófitas: o banco 01 correspondeu à Nymphaea L., erva flutuante fixa; o banco 02 , correspondeu à Ludwigia L., erva rasteira flutuante ou fixa; e o banco 03, à Polygonum ferrugineum Wedd., erva emergente (POTT; POTT, 2000). Sobre cada banco de macrófita foi colocado um quadrante $50 \times 50 \mathrm{~cm}$. O material incluso na área do quadrante foi coletado com o auxílio de uma tesoura de poda, retirando apenas as estruturas correspondentes a cada macrófita, como raízes, folhas e talos. O material foi acondicionado em sacos plásticos com uma porção de água e levado ao laboratório para lavagem e triagem.
No laboratório, sobre um recipiente plástico, com o auxílio de pincel, as estruturas das macrófitas foram lavadas em água corrente. A água retida no recipiente foi filtrada em três peneiras de diferentes aberturas de malha, que foram sobrepostas da maior para a menor, sendo a última a peneira granulométrica $(250 \mu \mathrm{m})$. $\mathrm{O}$ material concentrado foi acondicionado em frascos de vidro devidamente etiquetados e fixados com álcool 70\%.

Posteriormente, a triagem e a identificação foram realizadas utilizando microscópio esterioscópio (Lumen LM310BZ) e microscópio óptico (Zeiss Primo Star Binocular Microscope). Os insetos encontrados, tanto na fase imatura quanto na juvenil, foram identificados ao nível taxonômico de família, através de bibliografia específica, como: Paprocki et al. (2004); Passos et al. (2007); Pinho (2008); Mugnai et al. (2010); Segura et al. (2011). No fim desse processo, as amostras foram depositadas no Laboratório de Ecologia do Semiárido da Universidade do Estado da Bahia, DCH Campus VI. 


\section{Análise dos dados}

A partir da composição de táxons de macroinvertevrados, foi elaborado o diagrama de VENN. Em seguida, foi feita a contagem dos organismos amostrados e calculado o Índice de frequência de ocorrência, sendo as famílias identificadas classificadas como: constantes, presentes em mais de 50\% das amostras; acessórias, presentes em $25 \%$ a $50 \%$ das amostras; acidentais, presentes em menos de $25 \%$ das amostras (DAJOZ, 1983). Além disso, foi elaborado o gráfico com as abundâncias relativas referentes às famílias de macroinvertebrados aquáticos através do software R, versão 4.0.2 (2020-06-22), pacote vegan 2.56 (OKSANEN, 2019). Por fim, os índices bióticos foram calculados por meio do Índice Biological Monitoring Working Party System (BMWP') e Average Score per Taxa (BMWP-ASPT) (PARANÁ, 2003; SILVA et al., 2016).

As pontuações atribuídas às famílias de macroinvertebrados aquáticos para composição do BMWP' foram ordenadas em nove grupos, seguindo gradiente de menor a maior tolerância dos organismos quanto à poluição orgânica (PARANÁ, 2003). A cada família se fez corresponder uma pontuação que oscila de 10 a 1 , sendo que as famílias mais sensíveis à contaminação recebem as maiores pontuações, chegando a ordem decrescente até um, onde estão aquelas mais tolerantes (PARANÁ, 2003). A somatória das pontuações de cada táxon gera uma pontuação final que determina a qualidade da água do ambiente estudado. Para isso, a pontuação final foi comparada com uma tabela de classificação, onde se verificou em qual intervalo das sete classes a qualidade da água se encontra conforme o índice BMWP' (PARANÁ, 2003). Quanto maior a pontuação, mais íntegra a qualidade do ambiente aquático (BUSS et al., 2003).
Por fim, foi aplicado o índice BMWP-ASPT (SILVA et al., 2016), obtido a partir do valor de BMWP' dividido pelo número de famílias identificadas no banco amostral em análise, quanto maiores os valores do quociente do índice, mais elevada será a qualidade do ambiente aquático (WALLEY; HAWKES, 1997; SILVA et al., 2016).

\section{Resultados}

\section{Levantamento entomofaunístico}

No geral, a composição de macroinvertebrados identificados no presente estudo (Figura 2) apresentou valores de riqueza taxonômica e abundância total maiores na macrófita Ludwigia L., que apresentou 24 famílias e 4.288 org. $\mathrm{m}^{-2}$, representando $60 \%$ do total; seguida por Polygonum ferrugineum Wedd. com 18 famílias, 1.936 org. $\mathrm{m}^{-2}$ ou $27 \%$ e Nymphaea L. com 11 famílias e 896 org. $\mathrm{m}^{-2}$ ou $13 \%$ do total (Tabela 1).

Nota-se que a composição das 27 famílias de macroinvertebrados, que foram registradas, não ocorreu de forma similar em todas as macrófitas. Os dados revelaram que seis famílias desses organismos são exclusivas de Ludwigia L., duas são exclusivas de Polygonum ferrugineum Wedd., e apenas uma de Nymphaea L. (Figura 2). Além disso, observa-se que oito famílias são compartilhadas pelas três macrófitas, oito são comuns em Ludwigia L. e Polygonum ferrugineum Wedd., e duas são compartilhadas por Ludwigia L. e Nymphaea L.

Ao todo foram identificados 7120 espécimes pertencentes à Classe Insecta, sendo 33 táxons distribuídos em seis ordens e 27 famílias de insetos presentes nas três macrófitas amostradas: Coleoptera (7), Diptera (4), Ephemeroptera (3), Hemiptera (6), Odonata (4) e Trichoptera (3) (Tabela 1). 
FIGURA 2: Diagrama de VENN ilustrando as famílias de macroinverbrados exclusivas e compartilhadas por duas ou três espécies de macrófitas, Tanque de Arociras, Cactité, Bahia.

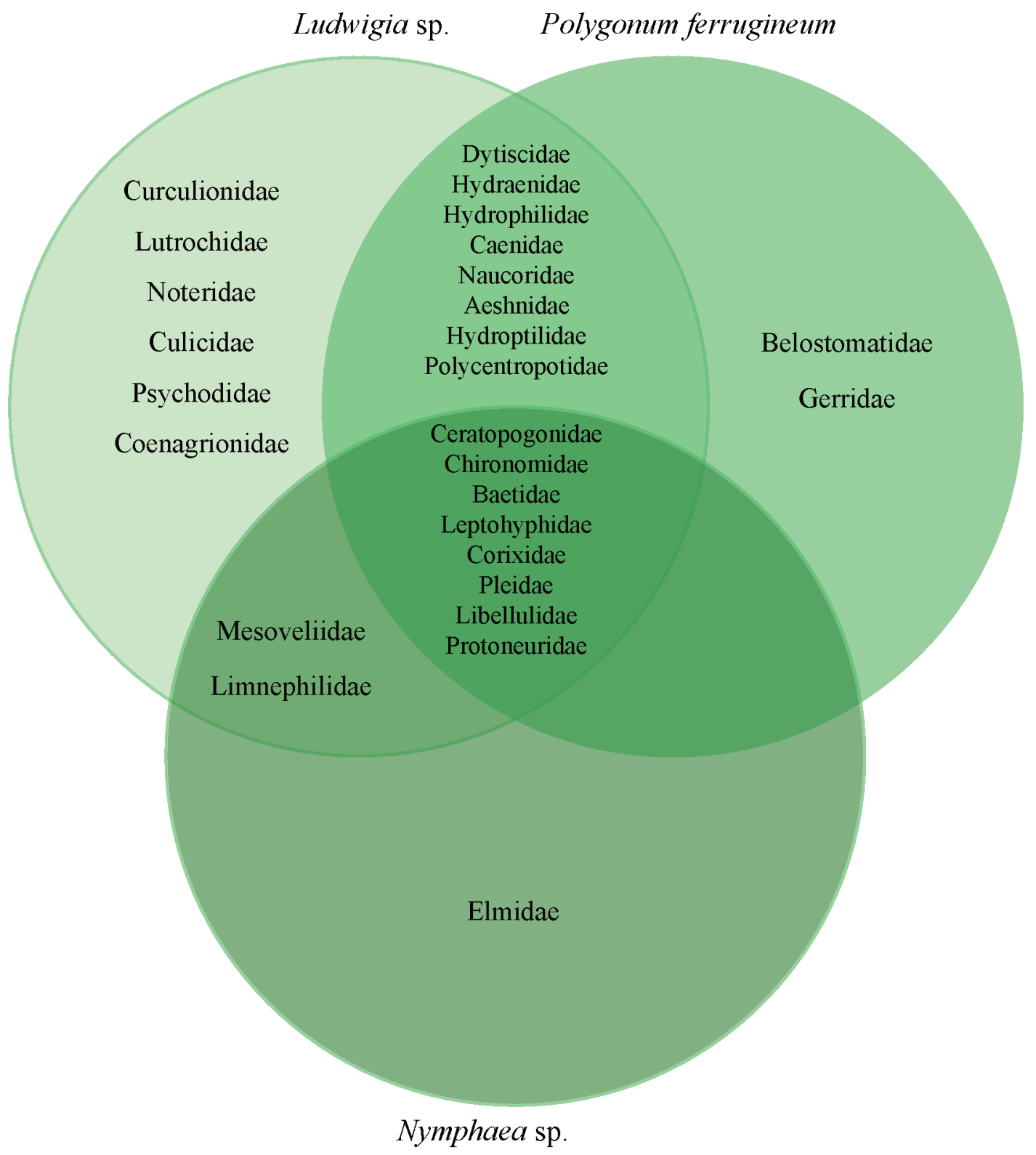


TABELA 1: Classificação taxonômica, riqueza, abundância (org.m $\left.{ }^{-2}\right)$, frequência de ocorrência (F.O\%) e classificação de Macroinvertebrados aquáticos presentes em macrófitas aquáticas (MAC), Tanque de Aroeiras, Caetité, BA.

\begin{tabular}{|c|c|c|c|c|c|c|}
\hline Táxons & $\begin{array}{l}\text { Nymphaea sp. } \\
\text { (MAC. 01) }\end{array}$ & $\begin{array}{l}\text { Ludwigia sp. } \\
\text { (MAC.02) }\end{array}$ & $\begin{array}{l}\text { Polygonum } \\
\text { ferrugineum } \\
\text { (MAC.03) }\end{array}$ & $\begin{array}{c}\text { Abundância/ } \\
\text { Família }\end{array}$ & F.O (\%) & Classificação \\
\hline
\end{tabular}

\section{Coleoptera}

Curculionidae Latreille, 1802

$\begin{array}{cc}- & 12 \\ - & 208 \\ 8 & - \\ - & 4 \\ - & 72 \\ - & 4 \\ - & 4\end{array}$

\section{Diptera}

Ceratopogonidae Newman, 1834

Chironomidae Newman, 1834

4

772

104

-
40
-
8
8
-
-
36
1252
-
-

Culicidae Stephens, 1829

Psychodidae Newman, 1834

\section{Ephemeroptera}

Baetidae Leach, 1815

Caenidae Newman, 1853

Leptohyphidae Edmunds e

Traver, 1954

$\begin{array}{cc}- & 116 \\ - & 4\end{array}$

\section{Hemiptera/Heteroptera}

Belostomatidae Leach, 1876

Corixidae Leach, 1815

16

16
$-\quad 340$

524

24

12

1252

12

33,33

Acessória

248

66,66

Constante

8

33,33

Acessória

12

66,66

Constante

80

66,66

Constante

4

33,33

Acessória

4

33,33

Acessória

Gerridae Leach, 1815

Mesoveliidae Douglas e Scott, 1867

Naucoridae Fallén, 1814

Pleidae Fieber, 1851

\section{Odonata}

Aeshnidae Rambur, 1842

Coenagrionidae Kennedy, 1920

Libellulidae Rambur, 1842

Protoneuridae Tillyard, 1917

\section{Trichoptera}

\begin{tabular}{lcccccc} 
Hydroptilidae Stephens, 1836 & - & 12 & 4 & 16 & 66,66 & Constante \\
Limnephilidae Kolenati, 1848 & 4 & 12 & - & 16 & 66,66 & Constante \\
Polycentropodidae Ulmer, 1903 & - & 8 & 36 & 44 & 66,66 & Constante \\
\hline Abundância total & 896 & 4.288 & 1.936 & 7.120 & & \\
\hline Riqueza taxonômica & 11 & 24 & 18 & & & \\
\hline
\end{tabular}


Entre as ordens de insetos aquáticos mais representativas, Diptera predominou sobre as demais em todas as macrófitas, apresentando maior abundância, detendo $62 \%$ dos organismos analisados, seguida por Ephemeroptera (18\%) e Hemiptera (9\%) (Tabela 1). Trichoptera apresentou menor abundância, representando apenas $1 \%$ dos organismos coletados.

Em relação às famílias encontradas, Chironomidae (Diptera) foi mais numerosa, apresentando maior dominância, representando $59 \%$ da abundância total, seguida pelas moscas Baetidae (9\%) e Caenidae (9\%) e pelo percevejo Pleidae (5\%) (Tabela 1; Figura 3). A família Chironomidae predominou nas três macrófitas amostradas, tendo maior destaque na macrófita Ludwigia L., com densidade de 2.144 org. $\mathrm{m}^{-2}$.

\section{Índices bióticos}

Na Tabela 2, consta a pontuação BMWP' por família em cada macrófita, por meio da qual é possível afirmar que Ludwigia L. apresentou uma maior quantidade de famílias sensíveis a fatores poluentes ou estressantes no ambiente, destacando-se as ordens Ephemeroptera, Odonata e Trichoptera com maior quantidade de famílias de organismos sensíveis, com três, quatro e três famílias, respectivamente. A macrófita Nymphaea L. teve uma menor abundância de organismos sensíveis, representados por seis famílias. As famílias com maior pontuação, ou seja, mais sensíveis, foram Aeshnidae e Libellulidae (Odonata). Por outro lado, as famílias que apresentaram menor pontuação, classificadas como mais tolerantes, foram Chironomidae e Culicidae (Diptera). A evidência desses organismos sensíveis e/ou tolerantes à poluição no Tanque de Aroeiras ainda requer novos estudos, sobretudo para testar se o aumento do espaçamento entre as macrófitas difere do método atual.

FIGURA 3: Abundância relativa das famílias de macroinvertebrados aquáticos presentes em macrófitas aquáticas, Tanque de Aroeiras, Caetité, BA.

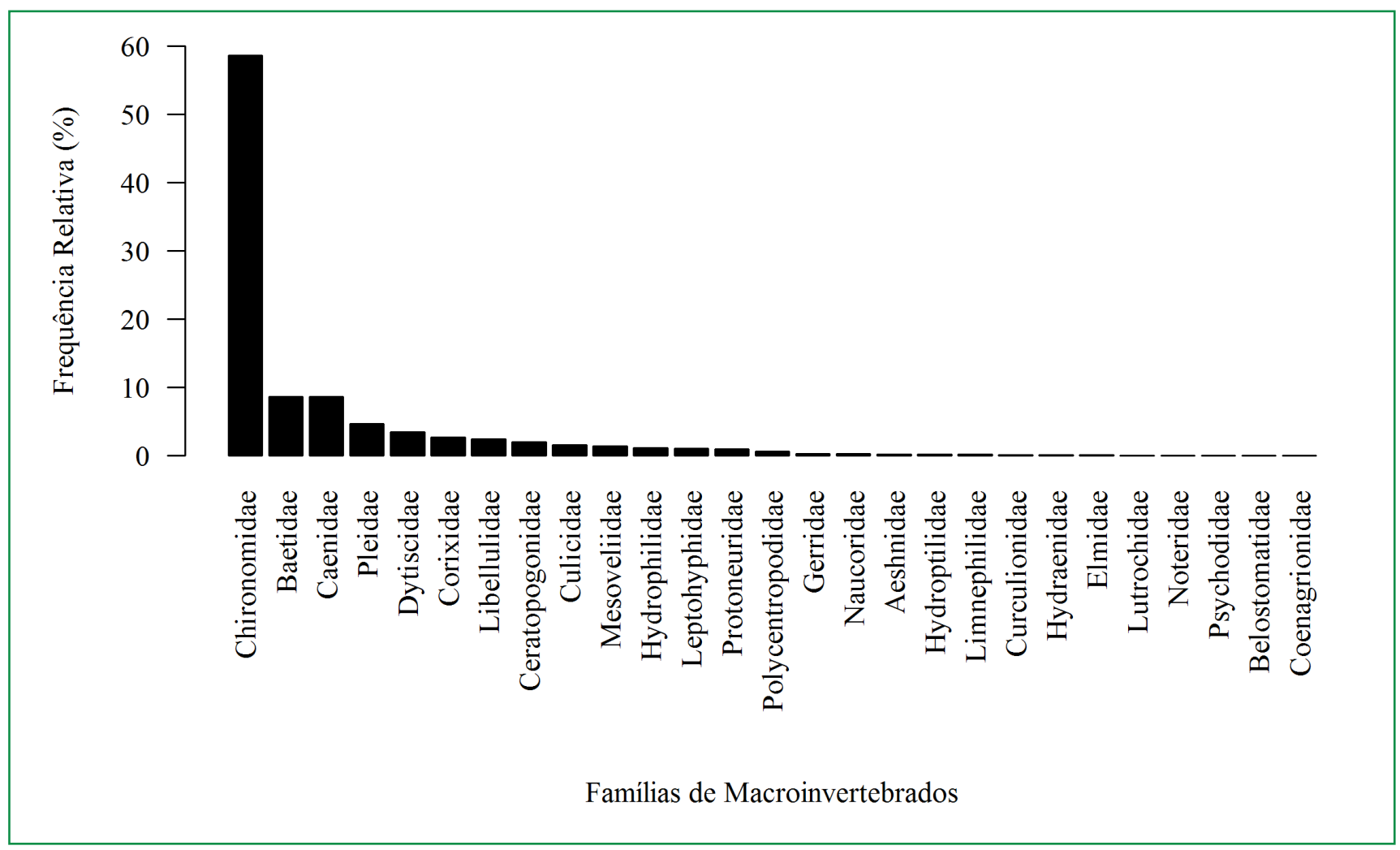


TABELA 2: Pontuação obtida do índice BMWP' (Biological Monitoring Working Party Score System) para as famílias de macroinvertebrados presentes em macrófitas aquáticas (MAC).

\begin{tabular}{|c|c|c|c|c|}
\hline \multirow[b]{2}{*}{ Táxons } & \multirow[b]{2}{*}{$\begin{array}{c}\text { Valores de } \\
\text { tolerância BMWP } *\end{array}$} & \multicolumn{3}{|c|}{ Pontuação BMWP'** } \\
\hline & & $\begin{array}{l}\text { Nymphaea sp. } \\
\text { MAC. } 01\end{array}$ & $\begin{array}{l}\text { Ludwigia sp. MAC. } \\
02\end{array}$ & $\begin{array}{c}\text { Polygonum } \\
\text { ferrugineum MAC. } \\
03\end{array}$ \\
\hline \multicolumn{5}{|l|}{ Coleoptera } \\
\hline Curculionidae & 5 & & $X$ & \\
\hline Dytiscidae & 4 & & $\mathrm{X}$ & $\mathrm{X}$ \\
\hline Elmidae & 6 & $\mathrm{X}$ & & \\
\hline Hydraenidae & 6 & & $X$ & $X$ \\
\hline Hydrophilidae & 4 & & $\mathrm{X}$ & $\mathrm{X}$ \\
\hline Lutrochidae & - & & $\mathrm{X}$ & \\
\hline Noteridae & - & & $\mathrm{X}$ & \\
\hline \multicolumn{5}{|l|}{ Diptera } \\
\hline Ceratopogonidae & 4 & $\mathrm{X}$ & $X$ & $\mathrm{X}$ \\
\hline Chironomidae & 2 & $\mathrm{X}$ & $\mathrm{X}$ & $\mathrm{X}$ \\
\hline Culicidae & 2 & & $\mathrm{X}$ & \\
\hline Psychodidae & 4 & & $\mathrm{X}$ & \\
\hline \multicolumn{5}{|l|}{ Ephemeroptera } \\
\hline Baetidae & 5 & $\mathrm{X}$ & $X$ & $X$ \\
\hline Caenidae & 5 & & $\mathrm{X}$ & $\mathrm{X}$ \\
\hline Leptohyphidae & 6 & $\mathrm{X}$ & $\mathrm{X}$ & $\mathrm{X}$ \\
\hline \multicolumn{5}{|l|}{ Hemiptera } \\
\hline Belostomatidae & - & & & $X$ \\
\hline Corixidae & 4 & $X$ & $X$ & $\mathrm{X}$ \\
\hline Gerridae & 4 & & & $\mathrm{X}$ \\
\hline Mesoveliidae & 4 & $X$ & & \\
\hline Naucoridae & 4 & & $\mathrm{X}$ & $\mathrm{X}$ \\
\hline Pleidae & 4 & $\mathrm{X}$ & $\mathrm{X}$ & $\mathrm{X}$ \\
\hline \multicolumn{5}{|l|}{ Odonata } \\
\hline Aeshnidae & 8 & & $X$ & $\mathrm{X}$ \\
\hline Coenagrionidae & 6 & & $X$ & \\
\hline Libellulidae & 8 & $X$ & $\mathrm{X}$ & $\mathrm{X}$ \\
\hline Protoneuridae & - & $\mathrm{X}$ & $X$ & $\mathrm{X}$ \\
\hline \multicolumn{5}{|l|}{ Trichoptera } \\
\hline Hydroptilidae & 7 & & $\mathrm{X}$ & $\mathrm{X}$ \\
\hline Limnephilidae & 7 & $X$ & $X$ & \\
\hline \multirow{2}{*}{ Polycentropodidae } & 7 & & $\mathrm{X}$ & $X$ \\
\hline & & 50 & 102 & 82 \\
\hline
\end{tabular}

* Sinal convencional (-): indica a ausência de valores de tolerância BMWP', conforme o score para a família no protocolo consultado (PARANÁ, 2003). ** O "X" presente nas colunas indica a presença da família de macroinvertebrados no ponto amostral da macrófita. 
O resultado do índice BMWP-APST, para as famílias de macroinvertebrados presentes nas macrófitas aquáticas amostradas, variou de 4,2 a 4,5 (Tabela 3). Apesar dessa variação na pontuação, nota-se que a categoria de qualidade de água do reservatório não sofreu diferenciação entre as macrófitas amostradas, sendo que, em todas, a água do Tanque de Aroeiras foi classificada como de provável poluição moderada.

TABELA 3: Resultado do índice biótico BMWP-ASPT para as famílias de macroinvertebrados aquáticos presentes em macrófitas aquáticas, indicando o nível de qualidade da água do Tanque de Aroeiras, Caetité-Bahia.

\begin{tabular}{lcc}
\hline Macrófitas & $\begin{array}{c}\text { Valor BMWP- } \\
\text { ASPT }\end{array}$ & Qualidade \\
\hline I: Nymphaea sp. & 4,5 & $\begin{array}{c}\text { Provável poluição } \\
\text { moderada }\end{array}$ \\
II: Ludwigia sp. & 4,2 & $\begin{array}{c}\text { Provável poluição } \\
\text { moderada }\end{array}$ \\
$\begin{array}{l}\text { III: Polygonum } \\
\text { ferrugineum }\end{array}$ & 4,5 & $\begin{array}{c}\text { Provável poluição } \\
\text { moderada }\end{array}$ \\
\hline
\end{tabular}

\section{Discussão}

\section{Levantamento entomofaunístico}

No Tanque de Aroeiras foram encontradas seis ordens e 27 famílias de insetos aquáticos. No estudo na represa do Ribeirão das Anhumas (SP), por meio de monitoramento realizado trimestralmente durante um ano, com amostragem em seis macrófitas distintas, foi verificada a ocorrência de sete ordens e 28 famílias pertencentes à classe Insecta (PEIRÓ; ALVES, 2006). Quanto à distribuição dos organismos, ao se comparar esses resultados com os encontrados no Tanque de Aroeiras, notou-se a ocorrência em comum de seis ordens e 17 famílias de insetos. Já na região semiárida paraibana, monitoramento sazonal com três subamostragens na Lagoa Panati, foi verificada a ocorrência de sete ordens e 32 famílias de insetos (ABÍLIO et al., 2007); comparando com os resultados do Tanque de Aroeiras, notou-se também a ocorrência em comum de seis ordens e 20 famílias.
As ordens que tiveram maior representação, seja por uma maior riqueza ou maior abundância, foram Coleoptera, Hemiptera e Diptera. As ordens Coleoptera e Diptera apresentam múltiplas adoções de estilos de vida aquáticos, fato que pode influenciar na riqueza e na abundância desses organismos. A ordem Hemiptera, entre os insetos hemimetábolos, possui maior diversidade em habitat aquáticos, podendo apresentar hábitos como capacidade de explorar o nêuston, superfície de águas lênticas, e também os que possuem asas podem migrar para lagos recém-formados, buscando condições favoráveis (GULLAN; CRANSTON, 2012). Entre os dípteros, a família Chironomidae foi dominante nos três bancos de macrófitas amostrados. A abundância e a dominância de Chironomidae associadas a macrófitas aquáticas em ambientes lênticos também foram registradas nos estudos de Peiró e Alves (2006), Albertoni et al. (2007), Henriques-de-Oliveira et al. (2007), Krawczyk et al. (2013) e Silva e Henry (2013).

A família Chironomidae ocorre majoritariamente em ambientes dulciaquícolas, possui ampla distribuição no mundo, sendo considerada a família mais numerosa de insetos aquáticos. Tal fato pode ser atribuído às adaptações desenvolvidas por suas larvas, permitindo que explorem e habitem todos os tipos de ambientes aquáticos, colonizando, basicamente, o sedimento e a vegetação aquática (CALLISTO et al., 2002; TRIVINHO-STRIXINO, 2014). A maior diversidade e densidade numérica de larvas de Chironomidae está relacionada a fatores climáticos, à menor profundidade da região litorânea, fazendo com que ocorra maior proliferação de algas perifíticas, à maior oferta de recursos alimentares e de matéria orgânica autóctone e alóctone, e às baixas concentrações de oxigênio do ecossistema (BUTAKKA et al., 2014; MACHADO et al., 2015). Nesse sentido, os insetos desenvolveram uma variedade de mecanismos eficientes para sobreviverem em baixas concentrações de oxigênio dissolvido, por exemplo: larvas de moscas (Diptera) utilizam sifões respiratórios terminais ou órgão torácico respiratório para obter oxigênio atmosférico e também para perfuração de raízes e caules de macrófitas, de onde retiram o oxigênio dos tecidos; as larvas de Chironomidae (apnêusticas) apresentam estratégias eficientes para trocas gasosas, como respiração cuticular potencializada por tubos, 
hemoglobina no fluido corpóreo e traqueias bem desenvolvidas (PINHO, 2008; GULLAN; CRANSTON, 2012; TRIVINHO-STRIXINO, 2014).

Em relação aos bancos de macrófitas amostrados, Ludwigia L. apresentou maior riqueza e abundância, seguida por Polygonum ferrugineum Wedd. e Nymphaea L. As características morfológicas de Ludwigia L. favoreceram a colonização por insetos, aumentando a riqueza e a abundância desses organismos. Essa macrófita apresentava caule rastejante e ramificado formando um banco, além de longas raízes e flutuadores esponjosos. Tais características aumentam a área de contato da macrófita com a água, ampliando a complexidade de habitat devido à morfologia e à textura da sua estrutura física. Esses aspectos, além de outros elementos estruturais, aumentam a heterogeneidade de habitat, possibilitando que os organismos encontrem proteção contra a predação, local de desova e alimento como perifíton e matéria orgânica particulada retida em suas raízes, folhas e caules (POI DE NEIFF; NEIFF, 2006; THOMAZ; CUNHA, 2010).

Além disso, a complexidade do habitat promovido pelas macrófitas influencia e fornece dependências distintas para as diferentes fases da vida de insetos associados, como os da família Libellulidae, provendo, para adultos, locais de oviposição e poleiros, e, para larvas, abrigo contra predadores e fontes de alimento (BRITO et al., 2021). Esses dados corroboram os encontrados por Silva e Henry (2013), que estudaram macroinvertebrados associados à Eichhornia azurea, em áreas marginais de ecossistemas lênticos. Os autores observaram que características da morfologia da planta, como hastes com raízes fasciculadas longas e folhas em contato com a água, aumentam a superfície de colonização por perifíton e retêm uma maior concentração de detrito e material orgânico particulado, suplemento alimentar desses organismos. Esses fatores também registrados em Ludwigia L. podem ter contribuído para o aumento e a manutenção da riqueza e da abundância dos macroinvertebrados. Albertoni et al. (2007) observaram também que Pistia stratiotes, planta flutuante, apresentou maior riqueza e abundância de macroinvertebrados, assim como Ludwigia L. no Tanque de Aroeiras.
Polygonum ferrugineum Wedd. apresentou riqueza e abundância em níveis intermediários em relação às outras macrófitas. No Tanque de Aroeiras, a macrófita formava um banco apresentando caules com partes submersas e partes aéreas, as folhas mais basais à haste tocavam a água e apresentava estágios iniciais de senescência. As macrófitas em estágios senescentes originam micro-habitat e aumentam nichos ecológicos, e são colonizadas durante a decomposição por bactérias e fungos, ampliando as concentrações de nitrogênio e proteína no substrato, liberando detrito. Tal fato torna o alimento mais atraente para macroinvertebrados e é um recurso importante para a cadeia detritívora (SILVA; HENRY, 2013).

Por outro lado, a macrófita Nymphaea L., diferentemente de Ludwigia L. e Polygonum ferrugineum Wedd., apresentou baixa riqueza e abundância taxonômica. Um fator importante que pode justificar essa condição é o enraizamento da macrófita de biótipo flutuante fixa, que diminui o aporte de material orgânico que entra no lago e se fixa em suas estruturas (POTT; POTT, 2000). Essa característica também foi registrada em lagos subtropicais, onde ocorreu uma menor riqueza e abundância em Nymphoides indica, planta enraizada, formada por longos pecíolos e por folhas flutuantes, banco escasso e pequeno aumento na exposição dos táxons associados a maiores variações abióticas (ALBERTONI et al., 2007).

Além dessas características estruturais das macrófitas que contribuem para a diversidade de habitat dos macroinvertebrados, destaca-se que esses organismos estão sujeitos a variadas condições nesses locais. Características como tipo de substrato e as variações físico-químicas são fatores determinantes que irão influenciar na sua adaptação (SALLES; FERREIRA-JÚNIOR, 2014).

Por outro lado, as macrófitas podem ser usadas para diferentes fins pelos macroinvertebrados, pois o crescimento dessas plantas contribui para a formação de micro-habitat, tornando os fatores físico-químicos menos restritivos, contribuindo para que a diversidade de insetos atinja níveis máximos em regiões litorâneas quando comparados a outras zonas (CALLISTO et al., 2001a; GULLAN; CRANSTON, 2012). Dessa forma, 
diferentes biótipos de macrófitas aquáticas podem ter influenciado na composição da comunidade de macroinvertebrados encontrada no Tanque de Aroeiras, visto que essas plantas ofertam diferentes condições de substrato, proteção e alimentação.

\section{Índices bióticos}

Entre as ordens de macroinvertebrados identificadas, organismos mais sensíveis e indicadores de água de boa qualidade, como os das ordens Trichoptera e Ephemeroptera, apresentaram uma abundância relativamente baixa quando comparada com a abundância daqueles mais tolerantes à poluição, que é o caso da ordem Diptera. Alguns indivíduos desse grupo, mais precisamente da família Chironomidae, foram registrados em abundância muito elevada nos três bancos de macrófitas amostrados, sendo um importante indicador de água de má qualidade.

Organismos da ordem Trichoptera e Ephemeroptera são típicos de ambientes limpos com águas oxigenadas e oligotróficas, podendo os organismos dessa última ordem, quando as condições ambientais não forem favoráveis, viverem em águas com baixos teores de oxigênio e altas temperaturas (CALLISTO et al., 2001b; ABÍLIO et al., 2007). Por outro lado, os organismos da família Chironomidae (Diptera) são considerados tolerantes, sobretudo porque: apresentam elevada capacidade adaptativa, como respirar pela superfície corpórea e ter pigmento hemoglobina; vivem em diferentes habitat, incluindo águas poluídas em condições quase anóxicas por longos períodos; além de serem bons indicadores de elevados teores de matéria orgânica no ambiente (PIEDRAS et al., 2006; GULLAN; CRANSTON, 2012; TRIVINHO-STRIXINO, 2014).

Dessa forma, a análise da qualidade da água do Tanque de Aroeiras, mesmo sendo uma avaliação instantânea em escala temporal, pode ser classificada como sendo de provável poluição moderada, conforme os dados obtidos pelos índice BMWP-ASPT para os bancos de macrófitas amostrados. Ressalta-se que, apesar de a confirmação dessa hipótese requerer um programa de biomonitoramento, os dados iniciais representam uma considerável evidência da degradação da qualidade da água desse ambiente. Afinal, algumas características dos macroinvertebrados, como mobilidade relativamente reduzida e longo ciclo, tornam esses organismos bons indicadores do que acontece ou aconteceu no sistema aquático (SANTOS, 2018).

Este trabalho é o primeiro levantamento de macroinvertebrados no Tanque de Aroeiras. Porém, desde 2013, nesse mesmo ambiente, estudo sobre a composição da biodiversidade aquática vem sendo desenvolvido. Dessa forma, espera-se que, a partir deste trabalho, estudos com maior robustez amostral e com métodos analíticos mais sofisticados possam auxiliar para melhor compreensão da estrutura e dinâmica dos macroinvertebrados, contribuindo para o conhecimento da ecologia aquática no semiárido brasileiro.

\section{Agradecimentos}

Ao Departamento de Ciências Humanas - Campus VI pelo apoio logístico, à Pró-Reitoria de Pesquisa e Ensino de Pós-Graduação (PPG) e à PRAES da Universidade do Estado da Bahia pela concessão da bolsa de iniciação científica PICIN e pelo apoio à participação do Congresso de Ecologia do Brasil, respectivamente; à Paloma Mendes Oliveira pelo acompanhamento e auxílio no início da pesquisa; à Marília Grazielly Mendes dos Santos pelo auxílio nas identificações das macrófitas aquáticas; e ao GPES, em especial, à LPEAQ, pelo apoio na realização deste estudo.

\section{Referências}

ABÍLIO, F. J. P.; RUFFO, T. L. M.; SOUZA, A. H. F. F.; FLORENTINO, H. S.; OLIVEIRA JUNIOR, E. H.; MEIRELES, B. N.; SANTANA, A. C. D. Macroinvertebrados bentônicos como bioindicadores de qualidade ambiental de corpos aquáticos da Caatinga. Oecologia Brasiliensis, Rio de Janeiro, v. 11, n. 3, p. 397-409, 2007.

AlBertoni, E. F.; PREllVITZ, L. J.; PALMA-SILVA, C. Macroinvertebrate fauna associated with Pistia stratiotes and Nymphoides indica in subtropical lakes (south Brazil). Brazilian Journal of Biology, São Carlos, v. 67, n. 3, p. 499-507, 2007.

BIUDES, J. F. V.; CAMARGO, A. F. M. Changes in biomass, chemical composition and nutritive value of Spartina alterniflora due to organic pollution in the Itanhaém River Basin (SP, Brazil). Brazilian Journal of Biology, São Carlos, v. 66, n. 3, p. 781-789, 2006. 
BRITO, J. S.; MICHELAN, T. S.; JUEN, L. Aquatic macrophytes are important substrates for Libellulidae (Odonata) larvae and adults. Limnology, Tokio, v. 22, n. 1, p. 139-149, 2021.

BUSS, D. F.; BAPTISTA, D. F.; NESSIMIAN, J. L. Bases conceituais para a aplicação de biomonitoramento em programas de avaliação da qualidade da água de rios. Cadernos de Saúde Pública, Rio de Janeiro, v. 19, n. 2, p. 465-473, 2003.

BUTAKKA, C. M. M.; GOMES, L. C.; TAKEDA, A. M. Taxonomic and numeric structure of Chironomidae (Diptera) in different habitats of a Neotropical floodplain. Iheringia, Série Zoologia, Porto Alegre, v. 104, n. 3, p. 314-322, 2014.

COLADELLO, L. F.; GALO; M. D. L. B. T.; SHIMABUKURO, M. H.; IVÁNOVÁ, I.; AWANGE, J. Macrophytes' abundance changes in eutrophicated tropical reservoirs exemplified by Salto Grande (Brazil): Trends and temporal analysis exploiting Landsat remotely sensed data. Applied Geography, Sevenoaks, v. 121, p. 102242, 2020.

CALliSTO, M.; GONÇALVES, J. F. Jr. A vida nas águas das montanhas. Ciência Hoje, Rio de Janeiro, v. 31, n. 182, p. 68-71, 2002.

CAllisto, M.; MORENO, P.; BARBOSA, F. A. R. Habitat diversity and benthic functional trophic groups at Serra do Cipó, Southeast Brazil. Revista Brasileira de Biologia, São Carlos, v. 61, n. 2, p. 259-266, 2001a.

CALLISTO, M.; MORENO, P.; GONÇALVES JR., J. F.; LEAL, J. J. F.; ESTEVES, F. A. Diversity and biomass of Chironomidae (Diptera) larvae in an impacted coastal lagoon in Rio de Janeiro, Brazil. Brazilian Journal of Biology, São Carlos, v. 62, n. 1, p. 77-84, 2002.

CALlisto, M.; MORETTI, M.; GOUlART, M. Macroinvertebrados bentônicos como ferramenta para avaliar a saúde de riachos. Revista Brasileira de Recursos Hídricos, Porto Alegre, v. 6, n. 1, p. 71-82, 2001 b.

DAJOZ, R. Ecologia Geral. 4 ed. São Paulo: Editora Vozes, 1983. $472 \mathrm{p}$.

FERREIRA-JÚNIOR, N.; SALLES, F. F.; HAMADA, N. Hexápodes aquáticos. In: HAMADA, N.; NESSIMIAN, J. L.; QUERINO, R. B. (Org.). Insetos aquáticos na Amazônia brasileira: taxonomia, biologia e ecologia. Manaus: Editora do INPA, 2014. p. 173-182.

FULAN, J. Â.; HENRY, R.; DAVANSO, R. Os efeitos da ação antrópica sobre a distribuição de macroinvertebrados no Rio Guareí, São Paulo. Estudos de Biologia, Curitiba, v. 34, n. 82, p. 51-56, 2012.

GULLAN, P. J.; CRANSTON, P. S. Os insetos: um resumo de entomologia. 4 ed. São Paulo: Roca, 2012. 496 p.

HENRIQUES-DE-OLIVEIRA, C.; BAPTISTA, D. F.; NESSIMIAN, J. L. Sewage input effects on the macroinvertebrate community associated to Typha domingensis Pers in a coastal lagoon in southeastern Brazil. Brazilian Journal of Biology, São Carlos, v. 67, n. 1, p. 73-80, 2007.

INMET - INSTITUTO NACIONAL DE METEOROLOGIA. Normais climatológicos. 2020. Disponível em <https://portal. inmet.gov.br/normais>.

JUNQUEIRA, V. M.; AMARANTE, M. C.; DIAS, C. F. S. Biomonitoramento da qualidade das águas da bacia do Alto Rio das Velhas através de macroinvertebrados. Acta Limnologica Brasiliensia, Rio Claro, v. 12, p. 73-87, 2000.

KLUMPP, A.; BAUER, K.; FRANZ-GERSTEIN, C.; DE MENEZES, M. Variation of nutrient and metal concentrations in aquatic macrophytes along the Rio Cachoeira in Bahia (Brazil). Environment International, Amsterdam, v. 28, n. 3, p. 165-171, 2002.

KRAWCZYK, A. C. D. B.; BALDAN, L. T.; ARANHA, J. M. R.; MENEZES, M. S.; ALMEIDA, C. V. The invertebrate's community in adjacent Alto Iguaçu's anthropic lakes of different environmental factors. Biota Neotropica, Campinas, v. 13, n. 1, p. 47-60, 2013.

MACHADO, N. G.; NASSARDEN, D. C. S.; SANTOS, F.; BOAVENTURA, I. C. G.; PERRIER, G.; SOUZA, F. S. C.; MARTINS, E. L.; BIUDES, M. S. Chironomus larvae (Chironomidae: Diptera) as water quality indicators along an environmental gradient in a neotropical urban stream. Revista Ambiente \& Água, Taubaté, v. 10, n. 2, p. 298-309, 2015.

MONTEIRO, T. R.; OLIVEIRA, L. G.; GODOY, B. S. Biomonitoramento da qualidade de água utilizando macroinvertebrados bentônicos: adaptação do índice biótico BMWP' à Bacia do Rio Meia Ponte-GO. Oecologia Brasiliensis, Rio de Janeiro, v. 12, n. 3, p. 553-563, 2008.

MORENO, P.; CALLISTO, M. Bioindicadores de qualidade de água ao longo da bacia do Rio das Velhas (MG). In. FERRACINI, V. L.; QUEIROZ, S. C. N.; SILVEIRA, M. P. (Ed.). Bioindicadores de qualidade de água. Jaguariúna: Embrapa, 2004. p. 95-116.

MUGNAI, R.; NESSIMIAN, J. L.; BAPTISTA, D. F. Manual de identificação de macroinvertebrados aquáticos do estado do Rio de Janeiro. 1 ed. Rio de Janeiro: Technical Books, 2010. 176 p.

OKSANEN, J. Vegan: ecological diversity. Processed with vegan 2.5-6 in R version 3.6.1 (2019-07-05) on August 31, 2019.

PAPROCKI, H.; HOLZENTHAL, R. W.; BLAHNIK, R. J. Checklist of the Trichoptera (Insecta) of Brazil. Biota Neotropica, Campinas, v. 4, n. 1, p. 1-22, 2004.

PARANÁ - SECRETARIA DO MEIO AMBIENTE E RECURSOS HÍDRICOS. Avaliação da qualidade da água através dos macroinvertebrados bentônicos: índice BMWP 2003. 2003. Disponível em <http://www.meioambiente.pr.gov.br/modules/ conteudo/conteudo.php? conteudo $=91>$.

PASSOS, M. I. S.; NESSIMIAN, J. L.; FERREIRA JR., N. Chaves para identificação dos gêneros de Elmidae (Coleoptera) ocorrentes no estado do Rio de Janeiro, Brasil. Revista Brasileira de Entomologia, Curitiba, v. 51, n. 1, p. 42-53, 2007.

PEIRÓ, D. F.; ALVES, R. G. Insetos aquáticos associados a macrófitas da região litoral da represa do Ribeirão das Anhumas (município de Américo Brasiliense, São Paulo, Brasil). Biota Neotropica, Campinas, v. 6, n. 2, p. 1-9, 2006.

PEREIRA NETO, M. C. Perspectivas da açudagem no semiárido brasileiro e suas implicações na região do Seridó Potiguar. Sociedade \& Natureza, Uberlândia, v. 29, n. 2, p. 285-294, 2017.

PIEDRAS, S. R. N.; BAGER, A.; MORAES, P. R. R.; ISOLDI, L. A.; FERREIRA, O. G. L.; HEEMANN, C. Macroinvertebrados bentônicos como indicadores de qualidade de água na Barragem Santa Bárbara, Pelotas, RS, Brasil. Ciência Rural, Santa Maria, v. 36, n. 2, p. 494-500, 2006. 
PINHO, L. C. Diptera. In: FROEHLICH, C. G. (Org.). Guia online: identificação de larvas de insetos aquáticos do estado de São Paulo. 2008. Disponível em <http://sites.ffclrp.usp.br/aguadoce/ guiaonline $>$.

POI DE NEIFF, A.; NEIFF, J. J. Riqueza de especies y similaridad de los invertebrados que viven en plantas flotantes de la Planicie de Inundación del Río Paraná. Interciência, Caracas, v. 31, n. 3, p. 220-225, 2006.

POTT, V. J.; POTT, A. Plantas aquáticas do Pantanal. 1. ed. Brasília: Embrapa, 2000. 404 p.

PRADO, D. E. As Caatingas da América do Sul. In: LEAL, I. R.; TABARELli, M.; SILVA, J. M. C. da (Org.). Ecologia e conservação da Caatinga. Recife: Ed. Universitária da UFPE, 2003. p. 3-74.

ROSENBERG, D. M.; RESH, V. H. Freshwater biomonitoring and benthic macroinvertebrates. 1 ed. New York: Chapman \& Hall, 1993. 483 p.

SALLES, F. F.; FERREIRA-JÚNIOR, N. Hábitat e hábitos. In. HAMADA, N.; NESSIMIAN, J. L.; QUERINO, R. B. (Org.). Insetos aquáticos na Amazônia brasileira: taxonomia, biologia e ecologia. Manaus: Editora do INPA, 2014. p. 39-50.

SANTOS, D. F. C. Índices bióticos como ferramentas de avaliação de riachos em uma região de Mata Atlântica. 2018. 43 f. Dissertação (Mestrado em Sistemas Aquáticos Tropicais) Universidade Federal de Santa Cruz, Ilhéus. 2018.

SEGURA, M. O.; VALENTE-NETO, F.; FONSECA-GESSNER, A. A. Chave de famílias de Coleoptera aquáticos (Insecta) do estado de São Paulo, Brasil. Biota Neotropica, Campinas, v. 11, n. 1, p. 393-412, 2011.

SILVA, C. V.; HENRY, R. Aquatic macroinvertebrates associated with Eichhornia azurea (Swartz) Kunth and relationships with abiotic factors in marginal lentic ecosystems (São Paulo, Brazil). Brazilian Journal of Biology, São Carlos, v. 73, n. 1, p. 149-162, 2013.
SILVA, K. W. S.; EVERTON, N. S.; MELO, M. A. D. Aplicação dos índices biológicos Biological Monitoring Working Party e Average Score per Taxon para avaliar a qualidade de água do rio Ouricuri no município de Capanema, estado do Pará, Brasil. Revista PanAmazônica de Saúde, Ananindeua, v. 7, n. 3, p. 13-22, 2016.

SILVEIRA-MANZOTTI, B. N.; MANZOTTI, A. R.; MÔNICA CENEVIVA-BASTOS, M.; CASATTI, L. Trophic structure of macroinvertebrates in tropical pasture streams. Acta Limnologica Brasiliensia, Rio Claro, v. 28, e15, 2016.

TANIGUCHI, H.; TOKESHI, M. Effects of habitat complexity on benthic assemblages in a variable environment. Freshwater Biology, Melville, v. 49, n. 9, p. 1164-1178, 2004.

THOMAZ, S. M.; CUNHA, R. B. The role of macrophytes in habitat structuring in aquatic ecosystems: methods of measurement, causes and consequences on animal assemblages' composition and biodiversity. Acta Limnologica Brasiliensia, Rio Claro, v. 22, n. 2, p. 218-236, 2010.

TRIVINHO-STRIXINO, S. Ordem Diptera, Família Chiromidae, guia de identificação de larvas. In. HAMADA, N.; NESSIMIAN, J. L.; QUERINO, R. B. (Org.). Insetos aquáticos na Amazônia brasileira: taxonomia, biologia e ecologia. Manaus: Editora do INPA, 2014. p. 457-660.

TUNDISI, J. G.; TUNDISI, T. M. Limnologia. São Paulo: Oficina de Textos, 2008. 632 p.

WALLEY, W. J.; HAWKES, H.A. A computer-based development of the biological monitoring working party score system incorporating abundance rating, site type and indicator value. Water Research, London, v. 31, n. 2, p. 201-210, 1997.

XAVIER, L. R. C. C.; SCHERNER, F.; BURGOS, D. C.; BARRETO, R. C.; PEREIRA, S. M. B. Urbanization effects on the composition and structure of macrophytes communities in a lotic ecosystem of Pernambuco State, Brazil. Brazilian Journal of Biology, São Carlos, v. 76, n. 4, p. 888-897, 2016. 